\title{
TWIN PREGNANCIES AND AORTIC INTIMA MEDIA THICKNESS: A NEW MODEL
}

\author{
S. Visentin ${ }^{1}$, E. Cosmi ${ }^{1}$, V. Zanardo ${ }^{2}$, D. Trevisanuto ${ }^{2}$, S. Vedovato ${ }^{2}$, F. Grumolato ${ }^{1}$ \\ ${ }^{I}$ Department of Gynecological Sciences and Human Reproduction, University of Padua, ${ }^{2}$ Pediatrics, Padua \\ University, Padua, Italy
}

Objective: Previously we showed that aorta intima-media thickness (aIMT) in IUGR fetuses with Doppler abnormalities is higher than in AGA. The aim of the present study is to validate this finding in twin pregnancies with the co-twin affected by IUGR or SGA.

Methods: Prospective longitudinal study has been performed in our University hospital between January 2009 and February 2011. IUGR co-twin was defined as fetuses with estimated fetal weight $(\mathrm{EFW})<10^{\text {th }}$ percentile with abnormal Doppler velocimetry (umbilical artery PI $>2$ SD) and SGA fetuses with EFW $<10^{\text {th }}$ percentile and normal Doppler velocimetry. aIMT was evaluated during routine third trimester ultrasonography.

Results: Sixty-three twin pregnancies were enrolled in the study. Among them 80 twins were AGA, 20 IUGR and 26 SGA. Median GA at birth was 35 weeks (range 32-36), and median birth weight $1665 \mathrm{~g}$ (range1246-1840), 2172 g (range 1838-2333), 2295 (range 1910-2550) in IUGR, SGA and AGA, respectively, showing a statistical difference between IUGR vs AGA $(p<0.0001)$ and vs SGA $(p=0.003)$. Median gestational age at aIMT measurements was 30 weeks (range 29-32). There was a significative difference in aIMT between IUGR, SGA and AGA.

Conclusions: aIMT is higher in IUGR compared to SGA vs AGA and in SGA vs AGA fetuses, highlighting that Doppler velocimetry and birth weight are predictors of aorta stiffness. Moreover, to our knowledge this is the first study considering aIMT in twin pregnancies, which may be considered a model of study to detect early cardiovascular markers in the same environment. 